\title{
Effect of different asthma treatments on risk of cold-related exacerbations
}

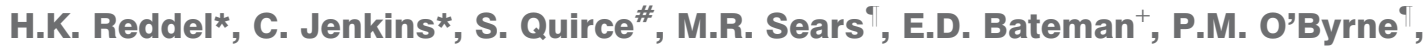

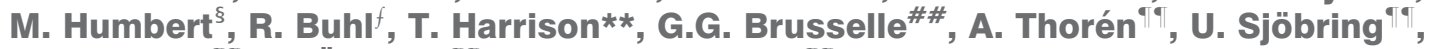

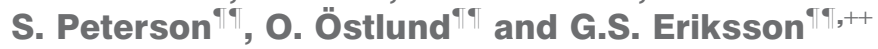

ABSTRACT: Common colds often trigger asthma exacerbations. The present study compared cold-related severe exacerbations during budesonide/formoterol maintenance and reliever therapy, and different regimens of maintenance inhaled corticosteroids (ICS), with or without long-acting $\beta_{2}$-agonists (LABA), and with as-needed short-acting $\beta_{2}$-agonists (SABA) or LABA.

Reported colds and severe exacerbations (defined by oral corticosteroid use and/or hospitalisation/emergency room visit) were assessed for 12,507 patients during 6-12 months of double-blind treatment. Exacerbations occurring $\leqslant 14$ days after onset of reported colds were analysed by a Poisson model.

The incidence of colds was similar across treatments. Asthma symptoms and reliever use increased during colds. Budesonide/formoterol maintenance and reliever therapy reduced severe cold-related exacerbations by $36 \%$ versus pooled comparators plus SABA (rate ratio (RR) 0.64 ; $p=0.002$ ), and for individual treatment comparisons, by $52 \%$ versus the same maintenance dose of ICS/LABA (RR 0.48; $p<0.001$ ); there were nonsignificant reductions versus higher maintenance doses of ICS or ICS/LABA (RR 0.83 and 0.72 , respectively). As-needed LABA did not reduce coldrelated exacerbations versus as-needed SABA (RR 0.96).

Severe cold-related exacerbations were reduced by budesonide/formoterol maintenance and reliever therapy compared with ICS with or without LABA and with as-needed SABA. Subanalyses suggested the importance of the ICS component in reducing cold-related exacerbations. Future studies should document the cause of exacerbations, in order to allow identification of different treatment effects.

KEYWORDS: Asthma, drug therapy, exacerbations, respiratory tract infections

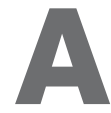
sthma is unusual amongst chronic diseases in that severe exacerbations occur even in mild or well-controlled disease. This paradox is largely attributable to viral respiratory infections causing clinical colds, which, although no more common in asthma [1], cause more prolonged and severe lower respiratory symptoms [1,2] and are responsible for $50-75 \%$ of asthma exacerbations in adults [3]. During confirmed viral infections, there is an influx of inflammatory cytokines and chemokines, and inflammatory cells, particularly neutrophils [2, 4]. As asthma is common and viral respiratory infections are ubiquitous, even a low rate of exacerbations in individual patients contributes substantially to the economic burden of asthma. This is reflected in recent guidelines with increasing focus on exacerbations and costs/risks of treatment as well as on patient-centred outcomes, such as symptoms and quality of life.

While the reduction in all-cause exacerbations with inhaled corticosteroids (ICS), alone or in combination with a long-acting $\beta_{2}$-agonist (LABA), is well established [5], little is known about effects on coldrelated exacerbations. In the past, exacerbations have been studied with ICS reduction [6] or allergen challenge models [7], but there is increasing recognition that viral exacerbations have different clinical and inflammatory features $[4,8,9]$. Therefore, the effect of pharmacological agents, including ICS, cannot be assumed to be the same for coldrelated and non-cold-related exacerbations.

Asthma clinical trials only rarely document causes of exacerbations and few include viral sampling, so information about therapeutic effects during
AFFILIATIONS

${ }^{*}$ Clinical Management Group, Woolcock Institute of Medical Research and University of Sydney, Sydney, NSW, Australia.

"Dept of Allergy, Hospital La Paz, Universidad Autónoma de Madrid, Madrid, Spain.

"Michael G. DeGroote School of Medicine, Faculty of Health Sciences, McMaster University, Hamilton, ON, Canada

+Division of Pulmonology, Dept of Medicine, University of Cape Town, Cape Town, South Africa.

${ }^{\text {s }}$ Service de Pneumologie et Réanimation Respiratoire, Hôpital Antoine-Béclère, Clamart, Université Paris-Sud 11, France.

'Pulmonary Dept, Mainz University Hospital, Mainz, Germany.

**Nottingham Respiratory Biomedical Research Unit, City Hospital Campus, Nottingham University, Nottingham, UK.

\#\#Dept of Respiratory Medicine, Ghent University Hospital, Ghent, Belgium.

"AstraZeneca Research and Development, and

${ }^{++}$Dept of Respiratory Medicine and Allergology, University Hospital, Lund, Sweden.

\section{CORRESPONDENCE}

H. K. Reddel, Woolcock Institute of Medical Research, PO Box M77, Missenden Road PO, NSW 2050 Australia

E-mail: hkr@med.usyd.edu.au

Received:

Dec 032010

Accepted after revision:

Feb 192011

First published online:

March 152011 
clinical colds is largely limited to in vitro rhinovirus studies [10-12]. However, adverse events (AEs), including clinical colds, are prospectively recorded at study visits with a standardised question such as "Have you had any health problems since your last visit?" Pooled AE records were recently used to demonstrate a reduced risk of cold-related exacerbations with ICS/LABA compared with ICS alone [13].

Clinical practice guidelines focus on reducing exacerbations, not only with maintenance therapy, but also by providing patients with written action plans [14]. Key components of effective action plans include increasing ICS doses after asthma worsens [15]. However, since the emergence of placebocontrolled evidence that doubling ICS doses does not reduce progression to exacerbation [16], guidelines currently recommend increasing short-acting $\beta_{2}$-agonist (SABA) doses for symptom relief, with no additional anti-inflammatory treatment unless the episode progresses to a severe exacerbation and oral corticosteroids (OCS) are required $[17,18]$; on average, this is $5-$ 10 days after asthma symptoms first start to increase [19, 20]. An alternative regimen, examined in recent large randomised controlled trials [21-25], uses budesonide/formoterol as maintenance therapy, with extra inhalations of budesonide/formoterol $(80 / 4.5$ or $160 / 4.5 \mu \mathrm{g})$ as needed for relief of asthma symptoms, regardless of their cause. The "action plan" for this treatment regimen thus includes an increase in ICS and rapidonset LABA, rather than SABA, as soon as symptoms and bronchoconstriction worsen. This regimen has been shown to reduce all-cause exacerbations with similar or better levels of asthma control than double-blind comparators [26], but its effect during colds has not been studied.

The present retrospective analysis, therefore, investigated the association between reported colds and severe asthma exacerbations amongst 12,507 patients who participated in five large clinical trials, and the extent to which different treatments prevented cold-related exacerbations.

\section{METHODS}

\section{Studies and population}

This retrospective analysis included five double-blind, randomised, parallel-group clinical studies of 6-12 months duration, running across summer and winter periods in the northern and southern hemispheres, investigating the efficacy of budesonide/ formoterol maintenance and reliever therapy (Symbicort SMART $_{\circledR}$ Turbuhaler ${ }^{\circledR}$; AstraZeneca AB, Lund, Sweden). Eligible patients were prescribed Global Initiative for Asthma (GINA) step 2-4 treatment before entry into the study, and had one or more asthma exacerbations in the previous year, bronchodilator reversibility, and suboptimally controlled asthma during run-in period. Time to the first severe exacerbation was the primary end-point for all studies. All studies were performed in accordance with the Declaration of Helsinki and Good Clinical Practice guidelines, and approved by independent ethics committees. All patients gave written informed consent. See the online supplement for further details (table E1).

For the primary analyses, pooled data from budesonide/ formoterol maintenance and reliever therapy, with reliever doses of $80 / 4.5$ or $160 / 4.5 \mu \mathrm{g}$, were compared with pooled data from three comparator regimens of fixed-dose maintenance treatment, namely higher maintenance dose ICS [21, 22], same-dose maintenance ICS/LABA [22, 23] and higher-dose maintenance ICS/LABA [24, 25], each with as-needed SABA (details and doses in online table E1). Similar analyses were also performed with each of the individual maintenance regimens.

For one study [23], data from a third treatment arm with samedose ICS/LABA plus as-needed formoterol $(4.5 \mu \mathrm{g}$; Oxis Turbuhaler, AstraZeneca $\mathrm{AB}$ ) were excluded from the main analyses, but a subanalysis comparing budesonide/formoterol maintenance and reliever therapy with same-dose ICS/LABA plus as-needed SABA or LABA was performed.

For seasonal analysis, each country was classified as northern or southern hemisphere, excluding Brazil, India, Indonesia, Malaysia, Philippines, Singapore, Thailand and Vietnam, which were defined as tropical countries; northern hemisphere "summer" was defined as March-August and "winter" as SeptemberFebruary, with the reverse for the southern hemisphere.

\section{Assessments}

Reported common colds

Reported common colds with onset after randomisation were captured by searching AEs, recorded at 3-monthly study visits, for the following preferred terms in MedDRA version 10 (Northrop Grumman Corporation, Los Angeles, CA, USA): upper respiratory tract infection (URTI); viral URTI; pharyngitis; viral pharyngitis; nasopharyngitis; and laryngopharyngitis. For each reported cold, the date of onset was defined as the "cold index day" and the following 14 days as the "cold period".

\section{Exacerbations}

A severe exacerbation was defined as deterioration in asthma resulting in OCS use for $\geqslant 3$ days and/or hospitalisation/ emergency room treatment. The prescription of OCS was according to the clinical judgement of the physician, who was unaware of the patient's (double-blind) treatment allocation. Milder exacerbations and those identified in two studies [21, 22] only by a fall in morning peak flow were excluded.

\section{Reliever use and asthma symptoms}

As-needed reliever use and asthma symptom score were recorded in patient diaries. Daily asthma symptom score (0-6) was the sum of night- and daytime scores (0: no symptoms; 3 : unable to perform normal activities or to sleep).

\section{Statistical methods}

All patients with recorded post-randomisation data were included in the analysis. Monthly rates of all reported colds and exacerbations were described by plots. As a stability check, similar plots were produced for the MedDRA diagnostic codes "conjunctivitis allergic", "rhinitis allergic" or "rhinitis seasonal", and for all noninfectious AEs, excluding allergy and asthma-related AEs.

The number of exacerbations in each season was analysed using a Poisson model for correlated within-patient observations with the factors, treatment, season, treatment-season interaction, hemisphere, hemisphere-season interaction and study, with (log-transformed) observation time as an offset.

The number of exacerbations for cold periods and non-cold periods was analysed using a Poisson model with the factors, 
treatment, cold status (cold period/non-cold period), treatment-cold status interaction, geographic area and study. Results were compared with a number of alternative models, including Cox models for time to exacerbation with season or cold status as time-dependent factors (see online supplement).

For patients with a reported cold, exacerbation incidence on days 0-14 after the patient's first reported cold was described by Kaplan-Meier plots and analysed using a Cox proportional hazard model for time from onset of cold to first exacerbation, which was censored after 14 days. For patients with a reported cold, with or without an exacerbation in the cold period, mean asthma symptom score and reliever use were plotted for days $-7-21$ relative to the first cold index day.

\section{RESULTS}

\section{Patient characteristics}

The analysis set comprised 12,507 patients ( $86 \%$ adults) with $66 \%$ from the northern hemisphere (table 1 and online table E2). Patients had characteristics of poorly controlled asthma at randomisation. Patients from different geographic areas showed some differences in baseline characteristics, including time from diagnosis, pre-entry LABA use and reliever use during the run-in period (table 1). However, baseline data were comparable between treatment groups (online table E2). Allergic conditions (rhinitis and/or conjunctivitis) were recorded as concomitant conditions at baseline for $22-34 \%$ patients (average $28 \%$ across studies) and were well matched between treatment groups.

\section{Incidence of reported colds}

The incidence of reported colds was driven almost exclusively by reports of URTI, pharyngitis and nasopharyngitis. Fewer colds were reported from northern hemisphere sites than southern and tropical sites $(0.29,0.39$ and 0.49 per patient per year, respectively). In the main analyses, rates and numbers of colds were similar across treatments, being reported by 20 $22 \%$ patients in 1-yr studies (table 2). Median duration of reported colds was 7 days (interquartile range 5-11 days).

\section{Reported colds, allergic episodes and all-cause exacerbations by season}

Reported colds and all-cause exacerbations displayed a similar seasonal pattern with winter peaks in both hemispheres, which were lower in northern- than southern-hemisphere countries (fig. 1a-d). Noninfectious AEs were stable throughout the year in both hemispheres (fig. 1e and f), with lower reporting rates from northern- compared with southern-hemisphere countries [27]. The incidence of allergic rhinitis and allergic conjunctivitis AEs was low compared with that of colds, with a different peak (northern: April/May; southern: October; fig. $1 \mathrm{~g}$ and $\mathrm{h}$ ). There were too few children/adolescents to examine seasonal patterns separately.

The rate of severe exacerbations was significantly lower with budesonide/formoterol maintenance and reliever therapy (9$13 \%)$, compared with pooled and individual fixed-dose maintenance treatments plus as-needed SABA (12-22\%) (table 2). During both summer and winter periods, significantly fewer exacerbations occurred with budesonide/formoterol maintenance and reliever therapy compared with pooled treatment comparators plus SABA (fig. 1 and online table E3; $\mathrm{p}<0.001$ ), and also compared with most individual treatment comparators (online fig. E1; all p $<0.001$ ). The exception was in the comparison with higher maintenance dose ICS/LABA plus SABA, where there was a significant reduction in all-cause exacerbations with budesonide/formoterol maintenance and reliever therapy during winter (41\%; rate ratio (RR) $0.59,95 \%$

TABLE 1 Demographic and baseline data

Pooled patient data ${ }^{\#}$

\begin{tabular}{cc} 
Randomised treatment \\
\hline All comparators $\quad \begin{array}{c}\text { Budesonide/Formoterol } \\
\text { maintenance plus reliever } \\
\text { therapy }\end{array}$
\end{tabular}

\begin{tabular}{lc}
\hline Patients n & 7281 \\
Males $\mathbf{n}(\%)$ & $3030(42)$ \\
Age yrs mean (range) & $39.0(4-83)$ \\
Entry ICS dose $\boldsymbol{\mu g}$ mean (range) & $709(100-3200)$ \\
Entry LABA use \% & 44 \\
Asthma diagnosis yrs median (range) & $11(0-77)$ \\
FEV $\mathbf{1} \%$ pred & $72.0 \pm 12.5$ \\
As-needed reliever use inhalations per day & $2.2 \pm 1.4$ \\
Total daily symptom score & $1.77 \pm 0.96$
\end{tabular}

Data are presented as mean \pm SD, unless otherwise stated. Pooled baseline and demographic data for patients receiving budesonide/formoterol maintenance and reliever therapy, and pooled fixed dose maintenance treatments plus short-acting $\beta_{2}$-agonists (SABA), and for all patients by geographic location. ICS: inhaled corticosteroid; LABA: long-acting $\beta_{2}$-agonist; FEV1: forced expiratory volume in $1 \mathrm{~s} ; \%$ pred: \% predicted. ${ }^{*}$ : the ICS/LABA plus formoterol as-needed arm in the study by RABE et al. [23] was not included in the main pooled analysis. For demographic data, for each of the different treatment groups, refer to the individual references (higher maintenance dose of ICS plus SABA [21, 22]; same maintenance dose ICS/LABA plus SABA [22, 23]; higher maintenance dose ICS/LABA plus SABA [24, 25]; Budesonide/formoterol maintenance and reliever therapy [21-25]). 


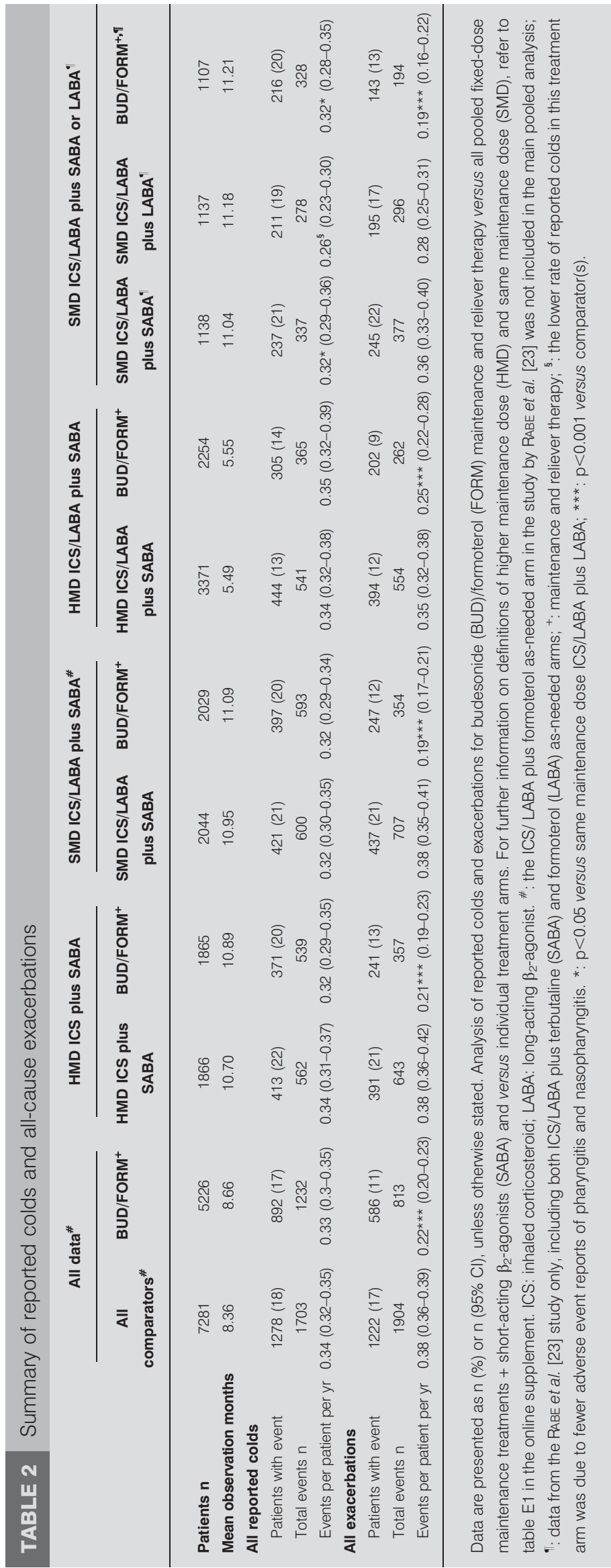

CI $0.46-0.76 ; \mathrm{p}<0.001)$ but no significant reduction during summer (14\%; RR 0.86, 95\% CI 0.63-1.18; p=0.35) (online fig. E1).

\section{Severe exacerbations following onset of a reported cold}

The proportion of patients with an exacerbation within the week before a reported cold was low $(<0.2 \%$ across all groups) but increased rapidly after the index day by approximately nine-fold (table 3 ), with $10 \%$ of severe exacerbations occurring during reported cold periods. During cold periods, budesonide/formoterol maintenance and reliever therapy significantly decreased the risk of severe exacerbations, with a $36 \%$ reduction compared with pooled fixed-dose maintenance treatments plus SABA (RR 0.64, 95\% CI 0.48-0.84). For individual treatment comparisons (table 3 ), the greatest reduction in cold-related exacerbations was with budesonide/ formoterol maintenance and reliever therapy compared with same maintenance dose ICS/LABA plus SABA (52\% reduction; RR $0.48,95 \%$ CI 0.31-0.72), with nonsignificant reductions in cold-related exacerbations with budesonide/formoterol maintenance and reliever therapy compared with higher maintenance dose ICS plus SABA (17\% reduction; RR 0.83, 95\% CI 0.49-1.40), and higher maintenance dose ICS/LABA plus SABA (28\%; RR 0.72, 95\% CI 0.45-1.15).

For time to first severe asthma exacerbation from the first cold index day, budesonide/formoterol maintenance and reliever therapy was also superior when compared with pooled fixeddose maintenance treatments plus SABA, and compared with same maintenance dose ICS/LABA plus SABA (figs 2 and 3).

In the comparison of cold versus non-cold periods, there was no significant difference in exacerbation rate reduction with budesonide/formoterol maintenance and reliever therapy relative to pooled comparators $36 \%$ relative reduction in cold periods versus $43 \%$ in non-cold periods; RR 1.12 , 95\% CI $0.83-$ $1.50 ; \mathrm{p}=0.47)$. Cold periods comprised only 2.7 weeks per patient per year, so there was limited power for individual treatment comparisons, but compared with high-dose ICS, the magnitude of reduction in exacerbations with budesonide/ formoterol maintenance and reliever therapy was smaller in cold periods than non-cold periods (17 versus $47 \%$; RR 1.56 , 95\% CI 0.91-2.69; $\mathrm{p}=0.11$ ).

For patients taking the same maintenance dose of ICS/LABA but different reliever medications (fig. 3 and online table E5), the comparison of exacerbation rate and time to first exacerbation showed that as-needed budesonide/formoterol was significantly better than either as-needed SABA or as-needed LABA during both cold and non-cold periods. For the comparison between as-needed SABA and as-needed LABA, there was no difference during cold periods in either exacerbation rate or time to first exacerbation (fig. 3 and online table E5), whereas during non-cold periods, there were significant differences favouring as-needed LABA (table E5).

\section{Reliever use and asthma symptoms in association with reported colds}

During reported colds, mean as-needed reliever use increased, particularly during severe exacerbations (fig. 4). For patients reporting a cold, those with a cold-related exacerbation had higher reliever use, averaged over the whole study, than those 

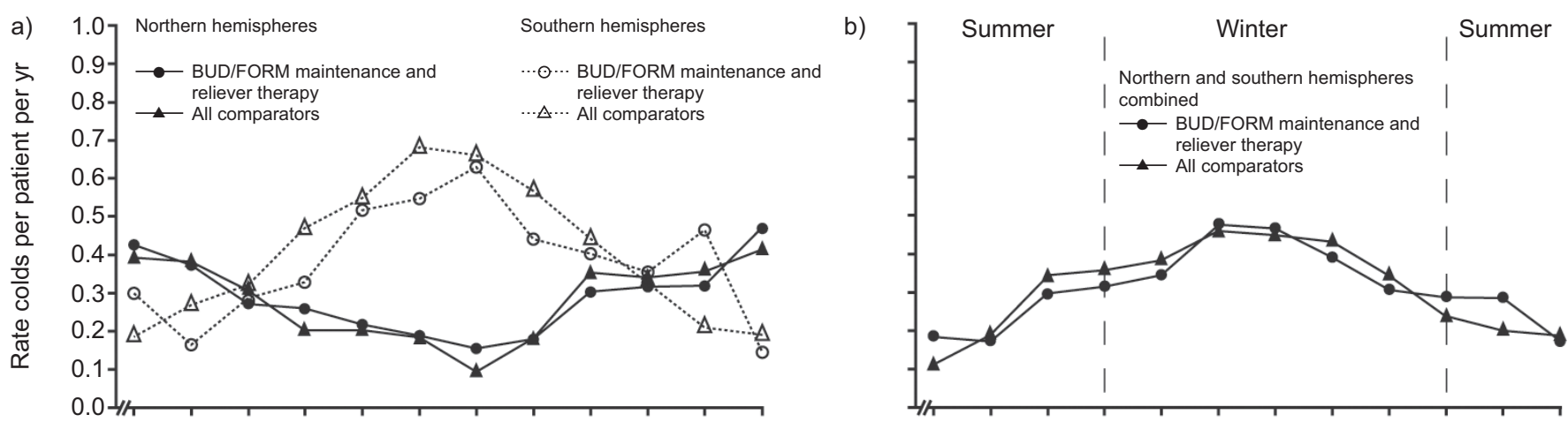

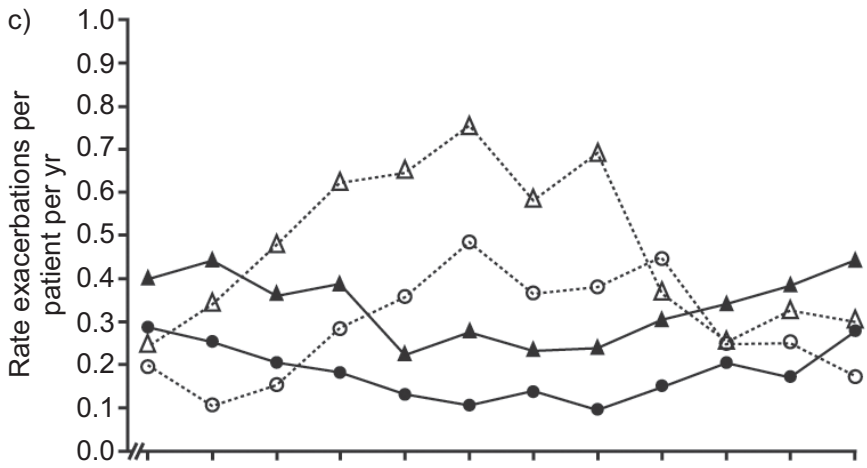

d)

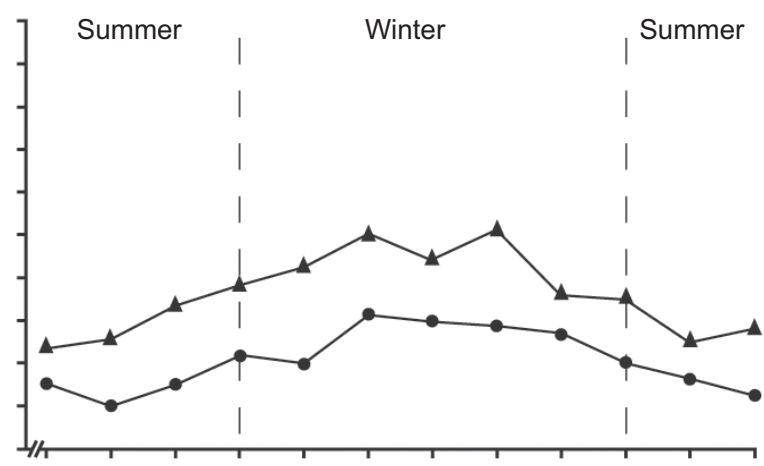

e) $\quad 1.0$
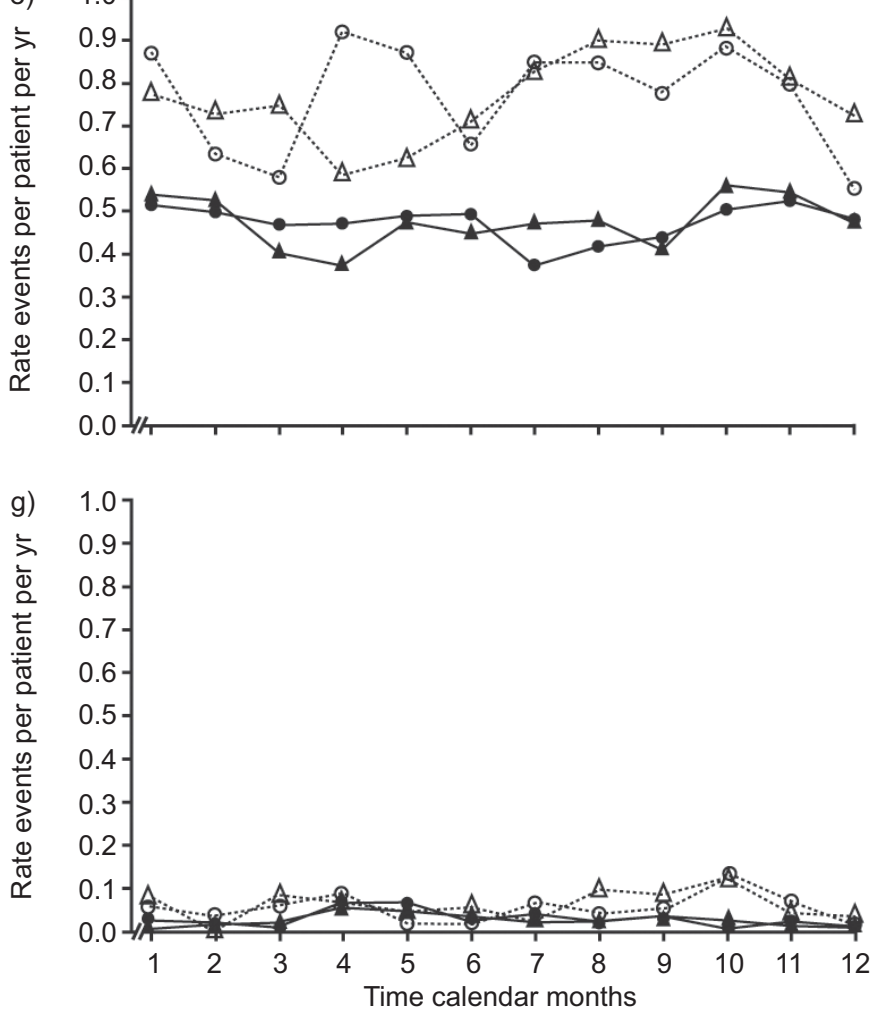

f)

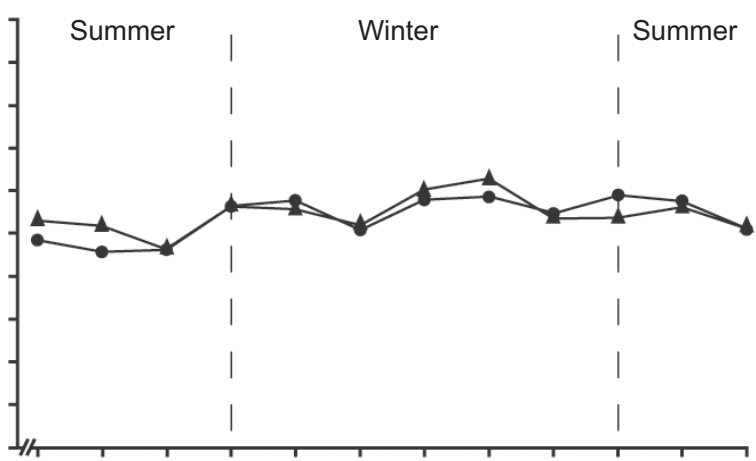

h)

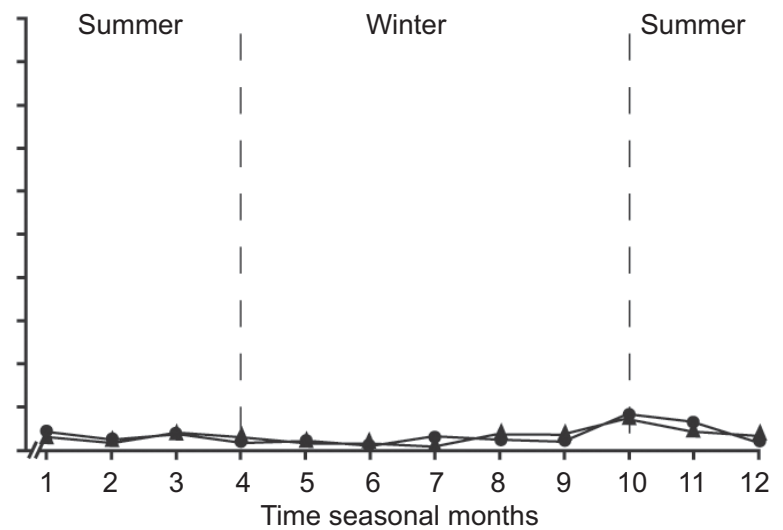

FIGURE 1. Annualised incidence (events per patient per year) of a, b) reported colds, c, d) all-cause exacerbations, e, f) noninfectious adverse events and g, h) allergic rhinitis or conjunctivitis, in all studies (budesonide (BUD)/formoterol (FORM) maintenance and reliever therapy versus pooled fixed-dose maintenance treatments plus shortacting $\beta_{2}$-agonist). a, c, e and g) Northern and southern hemispheres; b, $d, f$ and h) Seasonal month (southern hemisphere: 1 (January) to 12 (December); northern hemisphere: 1 (July) to 12 (June)). The inhaled corticosteroid/long-acting $\beta_{2}$-agonist plus formoterol as needed arm in the study by RABE et al. [23] was not included in these pooled analyses. 
without a post-cold exacerbation. Asthma symptoms showed a similar pattern (data not shown). Reliever use for the individual comparators is shown in online figure E2.

\section{DISCUSSION}

This retrospective analysis of a large clinical trial dataset confirmed that, even with highly effective treatment regimens, clinical colds remain an important trigger for severe asthma exacerbations, particularly during winter. Colds occurred at a similar rate with different controller treatment regimens, but budesonide/formoterol maintenance and reliever therapy reduced the risk of severe cold-related exacerbations by $36 \%$ compared with pooled comparator regimens in which the reliever medication was a SABA. The greatest difference in cold-related exacerbations (52\%) was seen in the comparison with same-dose maintenance ICS/LABA, where the only treatment difference was that, as symptoms increased, patients received additional ICS/LABA rather than additional SABA. These findings, together with those of other subgroup analyses, are consistent with current knowledge about the inflammatory milieu of the airways during viral infections and increasing evidence for the role of ICS dose in the management of worsening asthma.

Results from retrospective analyses should be interpreted with caution. In this study, viral causation of exacerbations was not proven, although strongly suggested by clinical diagnoses consistent with the common cold. No information was available about allergic sensitisation or allergen exposure, and even in prospective studies, as in clinical practice, it may be difficult to establish the relative contribution of viruses and allergen to individual exacerbations. AE data may be affected by patient recall or, as suggested by the geographic differences in the present study, by variation between clinical trial sites in the rigour with which they are recorded [27]. However, with $\mathrm{AE}$ data collected prospectively in randomised trials, bias, if any, is likely to be similar between groups. The rate of reported colds was lower than in prospective studies $(0.33-0.34$ versus 1.2-6.7 per patient per year [28]) and only $10 \%$ of severe exacerbations were during cold periods, suggesting that many associated colds were not reported or that the selected diagnostic codes lacked sensitivity. However, their specificity is supported by the winter peak in both hemispheres that contrasted with the spring-time peak for allergic rhinitis/ conjunctivitis, the contemporaneous increase in diary records for asthma symptoms and reliever use, and the marked increase in severe exacerbations after cold onset.

A strength of the present dataset was the opportunity to analyse cold-related exacerbations with four different treatment comparisons, and these analyses were instructive. While the halving of cold-related exacerbations with budesonide/ formoterol maintenance and reliever therapy compared with same maintenance dose of ICS/LABA plus as-needed SABA could potentially have been explained by the reliever's LABA component, this was not supported by a similar difference (54\%) between as-needed ICS/LABA and as-needed LABA, and the lack of evidence during colds for differences between

\section{TABLE 3 Exacerbation rates and treatment comparisons by cold status}

\begin{tabular}{|c|c|c|c|}
\hline & Mean ICS dose $\mu \mathrm{g} \cdot$ day $^{-1}$ & \multicolumn{2}{|c|}{ Exacerbation rate or ratio $(95 \% \mathrm{Cl})$} \\
\hline \multicolumn{4}{|l|}{ Pooled dataset ${ }^{\#}$} \\
\hline BUD/FORM maintenance and reliever therapy & 727 & $2.00(1.59-2.53)$ & $0.22(0.19-0.24)$ \\
\hline All fixed-dose maintenance treatments plus SABA & 987 & $3.15(2.66-3.74)$ & $0.38(0.35-0.41)$ \\
\hline BUD/FORM maintenance and reliever therapy versus all fixed-dose maintenance treatments plus SABA & & $0.64^{\circ}(0.48-0.84)$ & $0.57^{* \star *}(0.51-0.64)$ \\
\hline BUD/FORM maintenance and reliever therapy & 554 & $1.93(1.30-2.86)$ & $0.24(0.20-0.28)$ \\
\hline HMD ICS plus SABA & 1000 & $2.32(1.62-3.33)$ & $0.45(0.39-0.51)$ \\
\hline BUD/FORM maintenance and reliever therapy versus comparator & & $0.83^{+}(0.49-1.40)$ & $0.53^{* \star *}(0.44-0.64)$ \\
\hline \multicolumn{4}{|l|}{ BUD/FORM maintenance and reliever therapy versus SMD ICS/LABA plus SABA ${ }^{\#}$} \\
\hline BUD/FORM maintenance and reliever therapy & 582 & $1.99(1.41-2.80)$ & $0.20(0.17-0.24)$ \\
\hline SMD ICS/LABA plus SABA & 389 & $4.17(3.23-5.38)$ & $0.41(0.36-0.46)$ \\
\hline BUD/FORM maintenance and reliever therapy versus comparator & & $0.72^{\S}(0.45-1.15)$ & $0.70^{* * \star}(0.57-0.86)$ \\
\hline $\begin{array}{l}\text { Annualised exacerbation rate (exacerbations per yr) and the rate ratio for exacerbations for b } \\
\text { versus comparator(s) by cold status (cold period/non-cold period) using the statistical analysis } \\
\text { following onset of a reported cold. Cold periods comprised an average of } 2.7 \text { weeks of the tre } \\
\text { calculated as beclomethasone diproprionate-chlorofluorocarbon equivalent, based on Global In } \\
\text { period, including ICS delivered as maintenance and, where relevant, reliever therapy. SABA: } \\
\text { maintenance dose; LABA: long-acting } \beta_{2} \text {-agonist. }{ }^{\#} \text { : the ICS/LABA plus formoterol as needed } \\
\text { analysis but a comparison of the three different relievers is shown in online table E5; }{ }^{\bullet}: p=0.0\end{array}$ & $\begin{array}{l}\text { Udesonide (BUD)/formoterol } \\
\text { model (see Methods section) } \\
\text { eatment period per patient. N } \\
\text { hitiative for Asthma guidelines } \\
\text { short-acting } \beta_{2} \text {-agonist; HM } \\
\text { arm in the study by RABE et a } \\
02 ;{ }^{+}: p=0.49 ;{ }^{5}: p=0.17 ;{ }^{*}\end{array}$ & $\begin{array}{l}\text { (FORM) maintenance } \\
\text { A cold period was } d \\
\text { [17], over the whole } \\
\text { D: higher maintenan } \\
\text { [23] was not include } \\
\text { : }<0.001 \text {. }\end{array}$ & $\begin{array}{l}\text { and reliever therapy } \\
\text { fined as the } 14 \text { days } \\
\text { aroid (ICS) dose was } \\
\text { andomised treatment } \\
\text { e dose; SMD: same } \\
\text { d in the main pooled }\end{array}$ \\
\hline
\end{tabular}




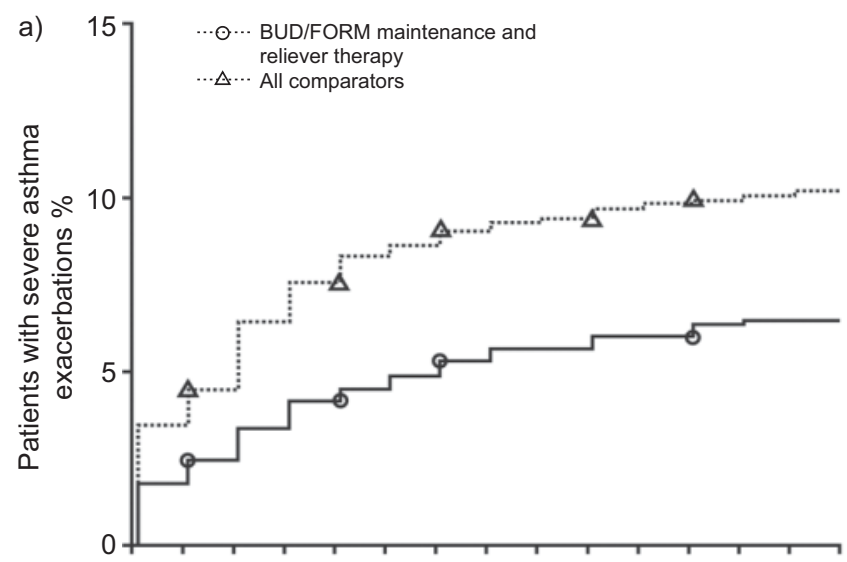

b)

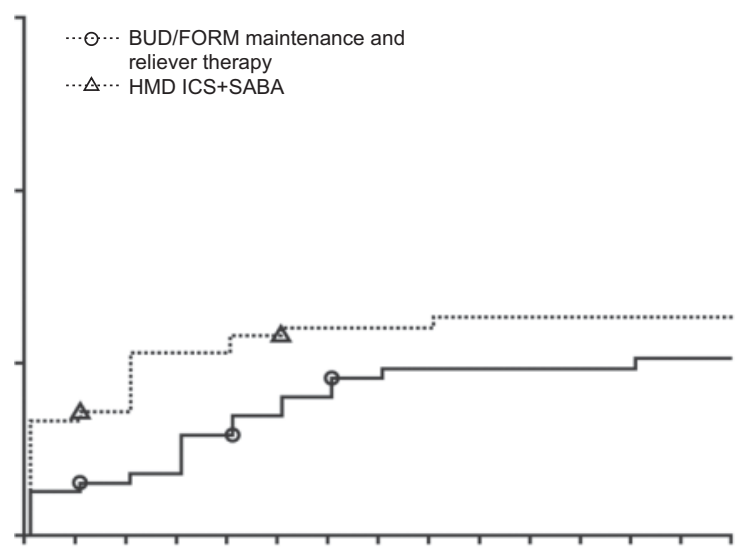

d)

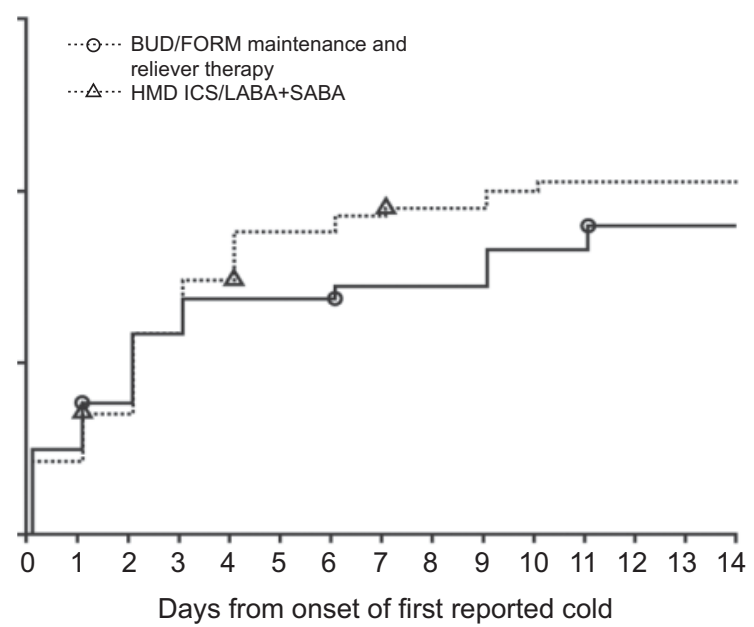

FIGURE 2. Time from onset of first reported cold to first severe asthma exacerbation. For patients reporting a cold, the figure shows Kaplan-Meier plots of time from cold index day (onset of first reported cold) to first severe asthma exacerbation for budesonide (BUD)/formoterol (FORM) maintenance and reliever therapy versus a) pooled fixeddose maintenance treatments plus short-acting $\beta_{2}$-agonists ( $\mathrm{SABA}$ ) (hazard ratio (HR) 0.60, 95\% Cl 0.43-0.82; $\mathrm{p}=0.0012$ ), b) higher maintenance dose (HMD) inhaled corticosteroids (ICS) plus SABA (HR 0.79, 95\% Cl 0.44-1.43; $p=0.43$ ), c) same maintenance dose (SMD) ICS/long-acting $\beta_{2}$-agonists (LABA) plus SABA (HR 0.31, 95\% CI 0.18-0.53; $p<0.001$ ) and d) HMD ICS/LABA plus SABA (HR 0.86, 95\% Cl 0.53-1.38; $p=0.52$ ). Only the first 14 days are shown. a, b) The ICS/LABA plus formoterol as needed arm in the study by RABE et al. [23] was not included in these pooled analyses; data from all three arms of that study are shown in figure 3.

as-needed LABA and SABA. However, a more consistent pattern was seen with ICS dose: the greatest difference in coldrelated exacerbations with budesonide/formoterol maintenance and reliever therapy (52\%) was seen versus same maintenance dose ICS/LABA (mean total beclomethasone dipropionate-chlorofluorocarbon-equivalent ICS dose 582 verus $389 \mu \mathrm{g}$ ), with smaller, nonsignificant differences as the relative ICS dose in the comparator arm increased $(28 \%$ reduction versus higher-dose ICS/LABA; 1,002 versus $1,341 \mu \mathrm{g})$ or doubled $(17 \%$ reduction versus higher-dose ICS alone; 554 versus 1,000 $\mu \mathrm{g})$. For most comparisons, the magnitude of the reduction in exacerbations with budesonide/formoterol maintenance and reliever therapy versus comparators was similar during cold periods and non-cold periods, with wider confidence limits reflecting the shorter observation period for cold periods ( 2.7 weeks per patient per year); however, the magnitude of the benefit from budesonide/formoterol maintenance and reliever therapy compared with higher-dose ICS was much lower during colds (17\%) than non-cold periods (47\%). Together, these findings suggest that the dose and/or timing of ICS are important for reducing coldrelated exacerbations.

Previous evidence for the protective effect of controller treatment during clinical colds has been largely inferred from all-cause exacerbations. Maintenance ICS reduce the risk of allcause exacerbations by $\sim 50 \%$ compared with placebo, with a greater reduction for higher versus lower ICS doses [5]. Adding LABA reduces all-cause exacerbations compared with ICS alone, although the advantage of adding LABA is smaller when the comparator is double-dose ICS [5]. By contrast with the present findings, increasing ICS dose after several days of worsening asthma is ineffective, except perhaps with a quadrupled dose [16].

However, cold-related and non-cold-related exacerbations may respond differently to treatment. Rhinovirus infection leads to rapid induction of multiple inflammatory cytokines and chemokines, and an influx of inflammatory cells (predominantly neutrophils) into the airway $[2,3]$. By contrast, exacerbations induced by ICS reduction are primarily characterised by 


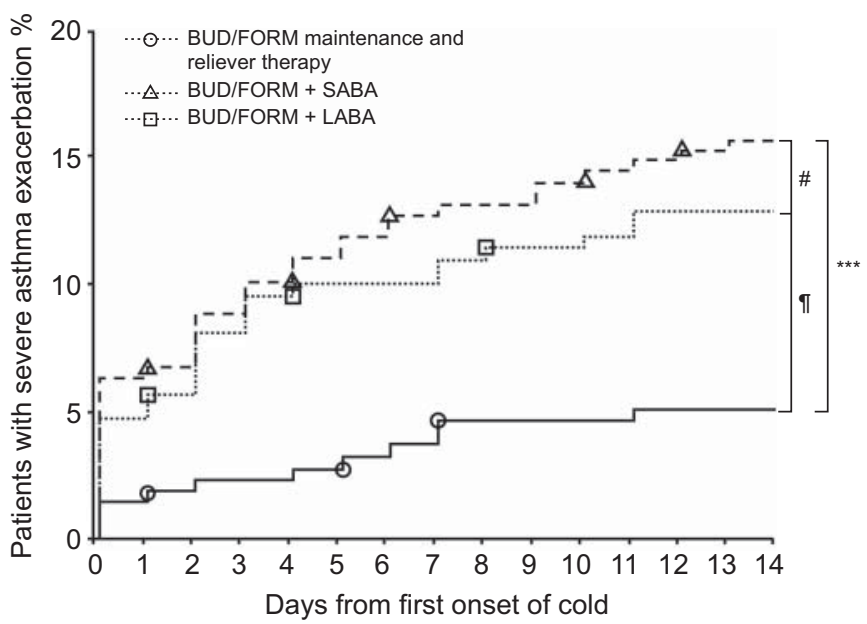

FIGURE 3. Kaplan-Meier plot of time from cold index day (onset of first reported cold) to first severe asthma exacerbation for budesonide (BUD)/formoterol (FORM) maintenance and reliever therapy versus same maintenance dose inhaled corticosteroids/long-acting $\beta_{2}$-agonists (LABA) (budesonide/formoterol) plus a short-acting $\beta_{2}$-agonist (SABA) (terbutaline) or plus LABA (formoterol). Only the first 14 days are shown. Budesonide/formoterol maintenance and reliever therapy versus budesonide/formoterol plus terbutaline as needed: hazard ratio (HR) 0.31 , 95\% Cl 0.16-0.60; budesonide/formoterol maintenance and reliever therapy versus budesonide/formoterol plus formoterol as needed: HR 0.38, 95\% Cl 0.19-0.76; budesonide/formoterol plus formoterol as needed versus budesonide/formoterol plus terbutaline as needed: HR 0.81; 95\% Cl: 0.49-1.33. * : $p=0.41 ;{ }^{\circ}: p=0.0066$; $\star * *: p<0.001$.

eosinophilic inflammation [6]. Likewise, in emergency department presentations, viral exacerbations are characterised by higher sputum neutrophils, lower lung function and a greater need for hospitalisation [4, 29], whereas nonviral exacerbations have sputum eosinophilia and better clinical outcomes [4]. In addition, cold-related exacerbations are associated with reduced diurnal variability and lack of response to SABA compared with poorly-controlled asthma [8].

Few studies in adults have examined the effect of long-term treatment on cold-related exacerbations, although several studies in infants and children have shown no reduction with regular ICS (e.g. [30]). In an experimental rhinovirus study in adults, 2 weeks of ICS pre-treatment failed to prevent virally induced airway inflammation [31]. In pooled clinical trials, fewer cold-related exacerbations occurred with salmeterol/ fluticasone than with fluticasone alone [13]. Further insights into the differences seen in the present analyses have been obtained from in vitro studies, which have shown a dose-dependent reduction in rhinovirus-induced interleukin (IL)-6, IL-8, C-C ligand (CCL)5 (a lymphocyte chemokine) and CXC ligand (CXCL)8 (a neutrophil attractor/activator) when airway epithelial cells were pre-treated with corticosteroids [10, 11, 32]. Pre-treatment with corticosteroids also abolished rhinovirusinduced loss of $\beta_{2}$-receptor function [33]. Salmeterol enhanced rhinovirus-induced IL-6 production [11], which may contribute to the adverse effects of LABA monotherapy [34], but salmeterol/fluticasone downregulated CCL5 and CXCL8 mRNA and protein expression at significantly lower concentrations than fluticasone alone, suggesting a synergistic effect [10].
Furthermore, rhinovirus-induced IL- 8 release was mediated by oxidative stress [35], suggesting a potential mechanism for relative corticosteroid resistance when rhinovirus-induced inflammation becomes established. Together, these previous studies indicate that ICS dose during viral infection, and the inclusion of LABA, may be important in preventing or managing cold-related exacerbations.

An additional factor is that patients taking conventional fixeddose maintenance treatment use SABA when symptoms worsen with a cold, often with no increase in anti-inflammatory treatment unless OCS are used. SABA may have pro-inflammatory effects [36-38], which are enhanced during rhinovirus infection [11], perhaps via atypical coupling, which may also reduce their bronchodilator effect [39]. Post-infection treatment of epithelial cells with budesonide and formoterol shows additive or synergistic suppression of rhinovirus-induced IL-6 and CCL5 [12]. Budesonide/formoterol maintenance and reliever therapy may provide protection not only by driving an early concurrent increase in ICS, but also by ensuring that virally induced inflammation is not further enhanced by unopposed use of SABA $[34,40]$. Given the rapid and intense inflammatory response to viral infection, further research is also needed to investigate potential action plan strategies for patients taking conventional fixed-dose maintenance ICS/LABA with as-needed SABA, for example, to evaluate the effectiveness of an immediate increase in ICS dose or short burst of OCS as soon as asthma begins to worsen with a cold rather than delaying additional anti-inflammatory treatment until increased symptoms have been present for $\sim 5$ days (as in previous studies of higher-dose ICS $[41,42]$ ) or have progressed to the extent that OCS would currently be recommended $[17,18]$. Although OCS are inexpensive and effective in the treatment of severe asthma exacerbations, concerns of the medical community $[43,44]$ and patients [45] about their side-effects indicate that the safety and cost-effectiveness of different action plan strategies should be evaluated in prospective studies in which all colds, cold-related exacerbations and medication side-effects are documented.

In future studies, it will be important to document the cause of asthma exacerbations rather than assume a uniform response. Detailed prospective studies are needed with nasopharyngeal and/or sputum sampling for viral identification and inflammatory characterisation. Routine use of a standardised cold questionnaire or even the simple question "Do you have a cold today?" [28] would increase our ability to identify and understand heterogeneity in asthma exacerbations and in therapeutic responses.

This retrospective study provides additional insight into strategies that may reduce the risk of cold-related asthma exacerbations, and supports the role of ICS dose and/or timing in this context. In patients with a history of previous exacerbations (a strong predictor of future exacerbations) the risk of coldrelated exacerbations can be halved by using budesonide/ formoterol as maintenance and reliever therapy, compared with same maintenance dose of ICS/LABA, with SABA as needed. There may be no significant differences in cold-related exacerbations with budesonide/formoterol maintenance and reliever therapy compared with higher maintenance dose ICS or ICS/ LABA, but the choice of regimen needs to take into account the costs and side-effects of long-term, high-dose treatment and the 

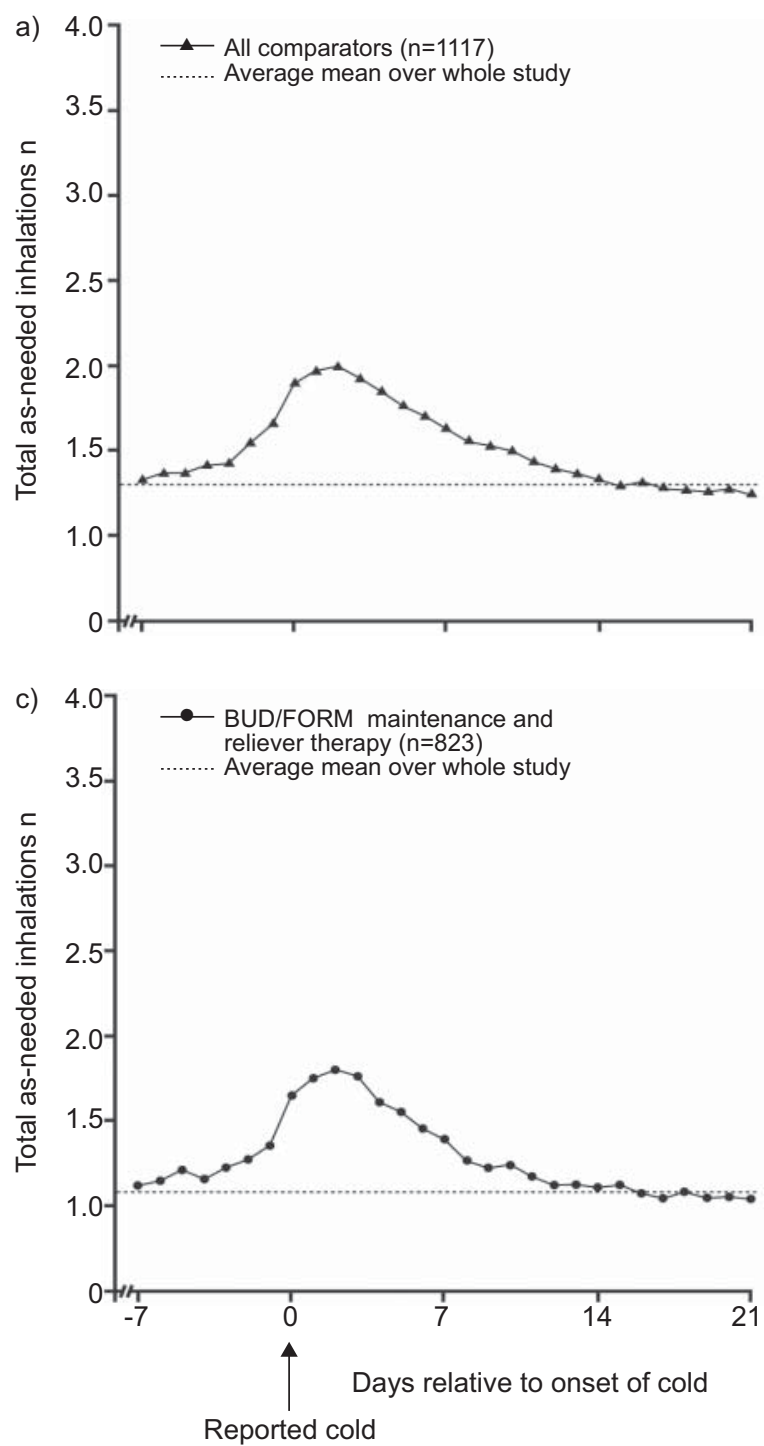

b)

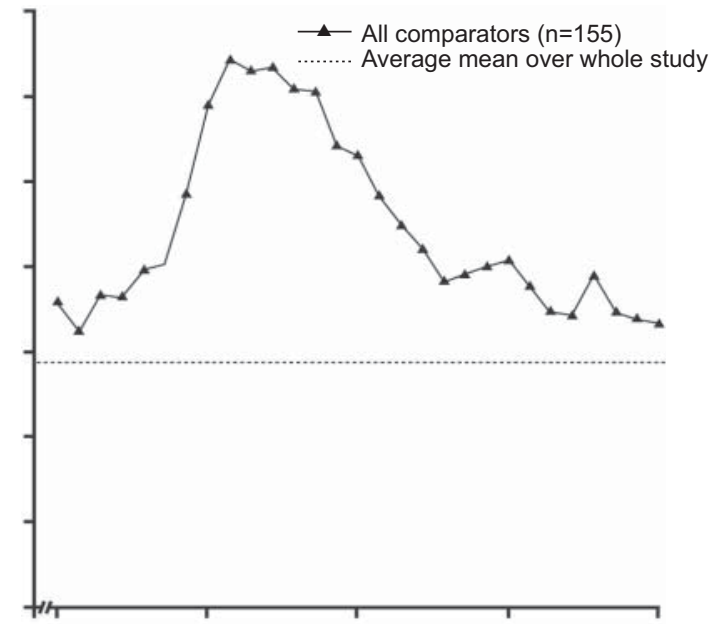

d)

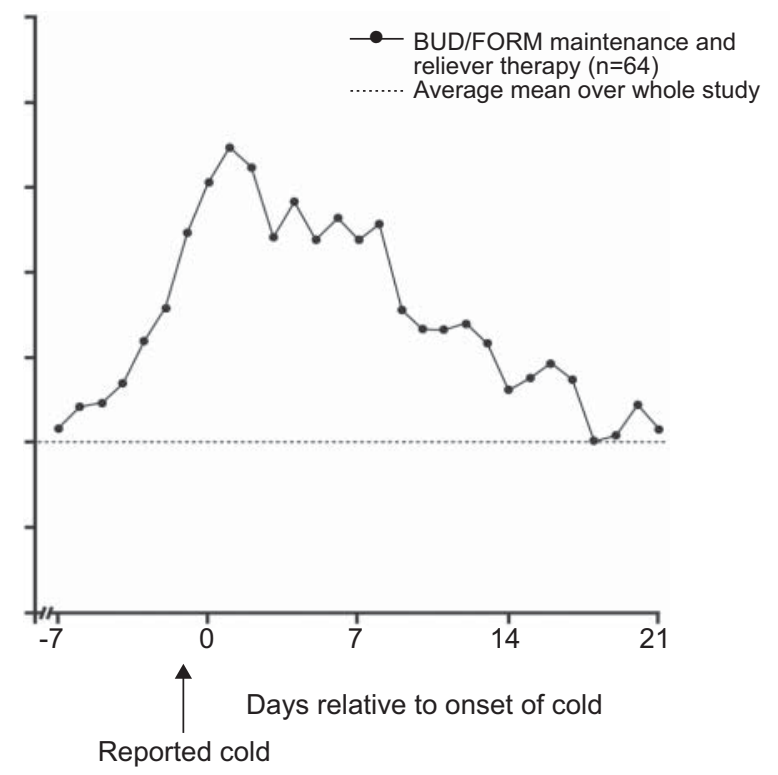

FIGURE 4. Reliever use associated with first reported cold. For patients reporting a cold, mean total daily reliever use (inhalations per patient per $24 \mathrm{~h}$ ) is shown over days -7-21 from the onset of first reported cold, from all studies, for a, b) all comparators and for c, d) budesonide (BUD)/formoterol (FORM) maintenance and reliever therapy. a, c) Patients reporting a cold only; b, d) patients reporting a cold and experiencing a severe exacerbation during the cold period. -----: on-treatment mean across the whole study for the same groups of patients.

potential risks of unopposed SABA treatment during viral infections, as well as patient preference. Prospective studies are needed to confirm the implications of these findings for management of worsening asthma during viral infections.

\section{SUPPORT STATEMENT}

The analysis was proposed separately by H.K. Reddel, G.G. Brusselle and A. Thorén, and was sponsored by AstraZeneca AB (Lund, Sweden).

\section{STATEMENT OF INTEREST}

Statements of interest for all authors and for the study itself can be found at www.erj.ersjournals.com/site $/ \mathrm{misc} /$ statements.xhtml

\section{ACKNOWLEDGEMENTS}

We thank J. Brennan (MediTech Media Ltd, Manchester, UK), who provided medical writing assistance on behalf of AstraZeneca AB. We thank G.P. Anderson (University of Melbourne, Melbourne, Australia) for critically evaluating the manuscript.

\section{REFERENCES}

1 Corne JM, Marshall C, Smith S, et al. Frequency, severity, and duration of rhinovirus infections in asthmatic and non-asthmatic individuals: a longitudinal cohort study. Lancet 2002; 359: 831-834.

2 Message SD, Laza-Stanca V, Mallia P, et al. Rhinovirus-induced lower respiratory illness is increased in asthma and related to virus load and Th1/2 cytokine and IL-10 production. Proc Natl Acad Sci USA 2008; 105: 13562-13567.

3 Jackson DJ, Johnston SL. The role of viruses in acute exacerbations of asthma. J Allergy Clin Immunol 2010; 125: 1178-1187.

4 Wark PAB, Johnston SL, Moric I, et al. Neutrophil degranulation and cell lysis is associated with clinical severity in virus-induced asthma. Eur Respir J 2002; 19: 68-75. 
5 Sin DD, Man J, Sharpe H, et al. Pharmacological management to reduce exacerbations in adults with asthma: a systematic review and meta-analysis. JAMA 2004; 292: 367-376.

6 Gibson PG, Wong BJO, Hepperle MJE, et al. A research method to induce and examine a mild exacerbation of asthma by withdrawal of inhaled corticosteroid. Clin Exp Allergy 1992; 22: 525-532.

7 Gauvreau GM, Evans MY. Allergen inhalation challenge: a human model of asthma exacerbation. Contrib Microbiol 2007; 14: 21-32.

8 Reddel H, Ware S, Marks G, et al. Differences between asthma exacerbations and poor asthma control. Lancet 1999; 353: 364-369.

9 Jayaram L, Pizzichini MM, Cook RJ, et al. Determining asthma treatment by monitoring sputum cell counts: effect on exacerbations. Eur Respir J 2006; 27: 483-494.

10 Edwards MR, Johnson MW, Johnston SL. Combination therapy: synergistic suppression of virus-induced chemokines in airway epithelial cells. Am J Respir Cell Mol Biol 2006; 34: 616-624.

11 Edwards MR, Haas J, Panettieri RA, et al. Corticosteroids and $\beta_{2}$ agonists differentially regulate rhinovirus-induced interleukin-6 via distinct cis-acting elements. J Biol Chem 2007; 282: 15366-15375.

12 Skevaki CL, Christodoulou I, Spyridaki IS, et al. Budesonide and formoterol inhibit inflammatory mediator production by bronchial epithelial cells infected with rhinovirus. Clin Exp Allergy 2009; 39: 1700-1710.

13 Prazma CM, Kral KM, Gul N, et al. Controller medications and their effects on asthma exacerbations temporally associated with upper respiratory infections. Respir Med 2010; 104: 780-787.

14 Gibson PG, Powell H, Coughlan J, et al. Self-management education and regular practitioner review for adults with asthma. Cochrane Database Syst Rev 2003; 1: CD001117.

15 Gibson PG, Powell H. Written action plans for asthma: an evidence-based review of the key components. Thorax 2004; 59: 94-99.

16 Quon BS, Fitzgerald JM, Lemiere C, et al. Increased versus stable doses of inhaled corticosteroids for exacerbations of chronic asthma in adults and children. Cochrane Database Syst Rev 2010; 10: CD007524.

17 Global Initiative for Asthma. Global Strategy for Asthma Management and Prevention. www.ginasthma.org/guidelinesgina-report-global-strategy-for-asthma.html Date last accessed: August 2010; Date last updated: 2010.

18 National Heart Lung and Blood Institute National Asthma Education and Prevention Program. Expert Panel Report 3: Guidelines for the Diagnosis and Management of Asthma. 2007. www.nhlbi.nih.gov/guidelines/asthma/asthgdln.htm Date last accessed: September 2007; Date last updated: August 2007.

19 Chan-Yeung M, Chang JH, Manfreda J, et al. Changes in peak flow, symptom score, and the use of medications during acute exacerbations of asthma. Am J Respir Crit Care Med 1996; 154: 889-893.

20 Tattersfield AE, Postma DS, Barnes PJ, et al. Exacerbations of asthma: a descriptive study of 425 severe exacerbations. The FACET International Study Group. Am J Respir Crit Care Med 1999; 160: 594-599.

21 Scicchitano R, Aalbers R, Ukena D, et al. Efficacy and safety of budesonide/formoterol single inhaler therapy versus a higher dose of budesonide in moderate to severe asthma. Curr Med Res Opin 2004; 20: 1403-1418.

22 O'Byrne PM, Bisgaard H, Godard PP, et al. Budesonide/ formoterol combination therapy as both maintenance and reliever medication in asthma. Am J Respir Crit Care Med 2005; 171: 129-136.

23 Rabe KF, Atienza T, Magyar P, et al. Effect of budesonide in combination with formoterol for reliever therapy in asthma exacerbations: a randomised controlled, double-blind study. Lancet 2006; 368: 744-753.

24 Kuna P, Peters MJ, Manjra AI, et al. Effect of budesonide/ formoterol maintenance and reliever therapy on asthma exacerbations. Int J Clin Pract 2007; 61: 725-736.
25 Bousquet J, Boulet LP, Peters MJ, et al. Budesonide/formoterol for maintenance and relief in uncontrolled asthma vs. high-dose salmeterol/fluticasone. Respir Med 2007; 101: 2437-2446.

26 Bateman ED, Reddel HK, Eriksson G, et al. Overall asthma control: the relationship between current control and future risk. J Allergy Clin Immunol 2010; 125: 600-608.

27 Joelson S, Joelson I-B, Wallander M-A. Geographical variation in adverse event reporting rates in clinical trials. Pharmacoepidemiol Drug Saf 1997; 3: S31-S35.

28 Walter MJ, Castro M, Kunselman SJ, et al. Predicting worsening asthma control following the common cold. Eur Respir J 2008; 32: 1548-1554.

29 Fahy JV, Kim KW, Liu J, et al. Prominent neutrophilic inflammation in sputum from subjects with asthma exacerbation. J Allergy Clin Immunol 1995; 95: 843-852.

30 Doull IJ, Lampe FC, Smith S, et al. Effect of inhaled corticosteroids on episodes of wheezing associated with viral infection in school age children: randomised double blind placebo controlled trial. BMJ 1997; 315: 858-862.

31 Grunberg K, Sharon RF, Sont JK, et al. Rhinovirus-induced airway inflammation in asthma: effect of treatment with inhaled corticosteroids before and during experimental infection. Am J Respir Crit Care Med 2001; 164: 1816-1822.

32 Suzuki T, Yamaya M, Sekizawa K, et al. Effects of dexamethasone on rhinovirus infection in cultured human tracheal epithelial cells. Am J Physiol Lung Cell Mol Physiol 2000; 278: L560-L571.

33 Trian T, Moir L, Qui G, et al. Rhinovirus-induced exacerbations of asthma - how is the $\beta_{2}$-adrenoceptor implicated? Am J Respir Cell Mol Biol 2010; 43: 227-233.

34 Johnston SL, Edwards MR. Mechanisms of adverse effects of $\beta$ agonists in asthma. Thorax 2009; 64: 739-741.

35 Biagioli MC, Kaul P, Singh I, et al. The role of oxidative stress in rhinovirus induced elaboration of IL- 8 by respiratory epithelial cells. Free Radic Biol Med 1999; 26: 454-462.

36 Aldridge RE, Hancox RJ, Taylor DR, et al. Effects of terbutaline and budesonide on sputum cells and bronchial hyperresponsiveness in asthma. Am J Respir Crit Care Med 2000; 161: 1459-1464.

37 Agarwal SK, Marshall GD Jr. Beta-adrenergic modulation of human type-1/type-2 cytokine balance. J Allergy Clin Immunol 2000; 105: 91-98.

38 Holden NS, Rider CF, Bell MJ, et al. Enhancement of inflammatory mediator release by $\beta_{2}$-adrenoceptor agonists in airway epithelial cells is reversed by glucocorticoid action. Br J Pharmacol 2010; 160: 410-420.

39 Anderson GP. Current issues with $\beta_{2}$-adrenoceptor agonists: pharmacology and molecular and cellular mechanisms. Clin Rev Allergy Immunol 2006; 31: 119-130.

40 Papi A, Caramori G, Adcock IM, et al. Rescue treatment in asthma. More than as-needed bronchodilation. Chest 2009; 135: 1628-1633.

41 FitzGerald JM, Becker A, Sears MR, et al. Canadian Asthma Exacerbation Study Group. Doubling the dose of budesonide versus maintenance treatment in asthma exacerbations. Thorax 2004; 59: 550-556.

42 Harrison TW, Oborne J, Newton S, et al. Doubling the dose of inhaled corticosteroid to prevent asthma exacerbations: randomised controlled trial. Lancet 2004; 363: 271-275.

43 Vestergaard P, Rejnmark L, Mosekilde L. Fracture risk associated with different types of oral corticosteroids and effect of termination of corticosteroids on the risk of fractures. Calcif Tissue Int 2008; 82: 249-257.

44 Kelly HW, Van Natta ML, Covar RA, et al. Effect of long-term corticosteroid use on bone mineral density in children: a prospective longitudinal assessment in the childhood Asthma Management Program (CAMP) study. Pediatrics 2008; 122: e53-e61.

45 Janson S, Becker G. Reasons for delay in seeking treatment for acute asthma: the patient's perspective. J Asthma 1998; 35: 427-435. 